\title{
Acute Extensive Anterior Wall Myocardial Infarction by ECG Finding
}

National Cancer Institute

\section{Source}

National Cancer Institute. Acute Extensive Anterior Wall Myocardial Infarction by ECG

Finding. NCl Thesaurus. Code C106496.

An electrocardiographic finding of patholog ic Q waves with accompanying ST elevation in leads $\mathrm{V} 1$ to $\mathrm{V} 6$, I and aVL, which is suggestive of acute myocardial infarction involving the anterior and anterolateral walls of the left ventricle. (CDISC) 